\title{
Pseudoblocks of Finite Dimensional Algebras
}

\author{
Afaf S. Alharthi ${ }^{1 *}$ and Ahmed A. Khammash ${ }^{1}$ \\ ${ }^{1}$ Department of Mathematical Sciences, Umm Al-Qura University, Makkah, Saudi Arabia \\ * Corresponding author
}

\begin{abstract}
The notion of pseudoblocks is borrowed from [1] and introduced to finite dimensional algebras. We determine the pseudoblocks for several known algebras such as the triangular algebra and the cyclic group algebra. Also, we determine the pseudoblocks for the group algebra of the special linear group $S L(2, p)$ in the natural characteristic being the only finite group of Lie type of finite representation type.
\end{abstract}

\section{Introduction}

In [1], the concept of pseudoblocks of the endomorphism algebra of a module over an algebra was introduced and shown to have a control on the (Brauer) block distribution of the simple modules for the endomorphism algebra in the light of the Brauer-Fitting correspondence. In this paper, we borrow the concept of pseudoblock from [1] to introduce it to finite dimensional (not only endomorphism) algebras. We investigate the pseudoblocks for several known algebras such as the triangular algebra and the cyclic group algebra. Towards the end, we investigate the pseudoblock distribution for the group algebra of the special linear group $S L(2, p)$ in the natural characteristic being the only finite group of Lie type of finite representation type.

\section{The pseudoblocks}

The Brauer-Fitting correspondence relates the isomorphism classes of indecomposable direct summands of a module to the projective indecomposable modules for its endomorphism algebra. This correspondence is shown in [1] to be incompatible with the (Brauer) block distribution of modules in both sides. Instead, the concept of the pseudoblock of an endomorphism algebra of a module over an algebra was introduced to ensure such compatibility. Here, we borrow this notion and introduce it for any finite dimensional algebra. Let $A$ be a finite dimensional algebra over an algebraically closed field $F, \bmod A$ denotes the category of finitely generated $A$-modules, and we write IndA for the class of indecomposable $A$-modules. We also write $(X, Y)_{A}$ for the $A$-homomorphism space $\operatorname{Hom}_{A}(X, Y)$ between two modules $X, Y \in \bmod A$. The pseudoblock linkage relation $\underset{P S A}{\approx}$ is an equivalence relation defined on IndA in terms of the homomorphism space.

Definition 2.1. If $X, Y \in$ IndA, then $X \underset{P S A}{\approx} Y$ iff there is a sequence of modules $X=X_{1}, X_{2}, \ldots, X_{t}=Y$ in IndA such that for all $i \in\{1,2, \ldots, t\}$ either $\left(X_{i}, X_{i+1}\right)_{A} \neq 0 \quad$ or $\quad\left(X_{i+1}, X_{i}\right)_{A} \neq 0$. 
Clearly, $\underset{P S A}{\approx}$ is an equivalence relation on IndA. We call the equivalence classes IndA/ $\underset{P S A}{\approx}$ are called pseudoblocks of the algebra $A$.

\section{Connection with the Brauer blocks}

The following shows that the pseudoblock linkage principle $\underset{P S A}{\approx}$ is stronger than the Brauer linkage principle $\underset{A}{\approx}$ relating indecomposable modules which belong to the same block.

Lemma 3.1. If $X, Y \in \operatorname{Ind} A$ and $X \underset{P S A}{\approx} Y$, then $X \underset{A}{\approx} Y$.

Proof. If $X \underset{P S A}{\approx} Y$, then there is a sequence of modules $X=X_{1}, X_{2}, \ldots, X_{t}=Y$ in IndA such that for all $i \in\{1,2, \ldots, t\}$ either $\left(X_{i}, X_{i+1}\right)_{A} \neq 0$ or $\left(X_{i+1}, X_{i}\right)_{A} \neq 0$. But this implies (see [2], p.93) that for all $i \in\{1,2, \ldots, t\}$ either $X_{i} \approx \underset{A}{\approx} X_{i+1}$ or $X_{i+1} \approx X_{i}$, and so $X \underset{A}{\approx} Y$.

Remark 3.2. The converse of lemma 3.1 does not hold. If we take $A=F S L(2,4)$ and CharF $=2$, then A has four simple modules namely $1,2_{1}, 2_{2}, 4$ (the latter being the Steinberg module) distributed into two Brauer blocks $\underbrace{1,2_{1}, 2_{2}}_{B_{1}}, \underbrace{4}_{B_{2}}$. The two 21 indecomposable modules 1, $1 \in$ IndA belong to the same (Brauer) block, but they lie in a two different pseudoblocks of A. To see this, $2_{2}$

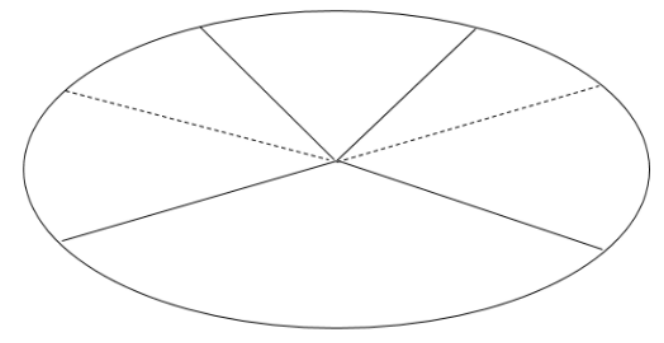

Figure 3.1: Some blocks in IndA split into union of pseudoblocks

It follows that, in principle, some (Brauer) blocks of A split into a union of pseudoblocks, and so we have $\mid$ IndA $/ \underset{A}{ } \mid \leqslant$ $|\operatorname{IndA} / \underset{P S A}{\approx}|$.

Motivation 3.3. If we take $Y \in \operatorname{modA}$ (not necessary indecomposable) and write Inds $(Y)$ for the isomorphism class of indecomposable A-summands of $Y$, then applying the linkage relation $\underset{P S A}{\approx}$ on Inds $(Y)$, it was shown in [1] that the (Brauer) block distribution of the simple modules of the endomorphism algebra $E(Y)=E_{n} d_{A}(Y)$ is controlled by the pseudoblocks distribution of Inds $(Y)$; that is if $Y_{i}, Y_{j} \in \operatorname{Ind}(Y)$ and $S_{i}, S_{j} \in \operatorname{Irr}(E(Y))$ are the corresponding simple E $(Y)$-modules under the Brauer-Fitting correspondence, then $S_{i} \underset{E(Y)}{\approx} S_{j} \Leftrightarrow Y_{i} \underset{P S A}{\approx} Y_{j}$.

A Useful Criterion 3.4. The pseudoblock equivalence relation $\underset{P S A}{\approx}$ is defined in terms of the homomorphism space $(X, Y)_{A}$. If $X, Y \in \operatorname{IndA}$, then $(X, Y)_{A} \neq 0$ if and only if $\exists K \leq_{A} X: X / K \cong$ submodule of $Y$. For, if $0 \neq f \in(X, Y)_{A}$, then $K=k e r f \supsetneqq X$ and $X / K \cong \operatorname{Imf} \leqslant_{A} Y$. Conversely, if $\exists K \leqslant_{A} X: X / K \cong T \leqslant_{A} Y$, then composing the map $X / K \cong T \longrightarrow Y$ with the natural map $X \rightarrow X / K$ we get a nonzero map $\theta: X \rightarrow Y$. Therefore, we have the figure 3.2

Lemma 3.5. $(X, Y)_{A} \neq 0$ if and only if $\exists K \leqslant_{A} X: X / K \cong$ a submodule of $Y$.

\section{Connection with tensor algebras}

Suppose that $A_{1}, A_{2}$ are two finite dimensional $F$-algebras. If $X_{i} \in \operatorname{Ind}\left(A_{i}\right) ; i=1,2$, then it is known (by considering endomorphism algebras) that $X_{1} \otimes X_{2} \in \operatorname{Ind}\left(A_{1} \otimes A_{2}\right)$. The following theorem shows that the concept of pseudo-blocks is compatible with tensor operation of modules.

Theorem 4.1. [3]. If $X_{i}, X_{i}^{\prime} \in \operatorname{Ind}\left(A_{i}\right) ; i=1,2$, then $X_{1} \otimes X_{2} \underset{P S\left(A_{1} \otimes A_{2}\right)}{\approx} X_{1}^{\prime} \otimes X_{2}^{\prime}$ if and only if $X_{1} \underset{P S A_{1}}{\approx} X_{1}^{\prime} \wedge X_{2} \underset{P S A_{2}}{\approx} X_{2}^{\prime}$. 


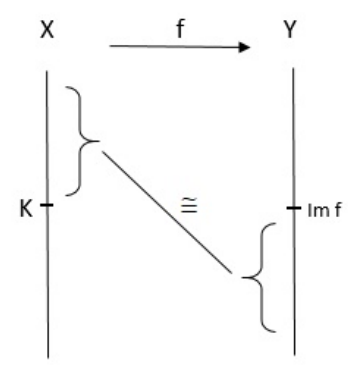

Figure 3.2

Proof. Since $X_{1} \underset{P \widetilde{S A}_{1}}{\approx} X_{1}^{\prime}$, there is a sequence $X_{1}=U_{1}, U_{2}, \ldots, U_{t}=X_{1}^{\prime}$ in $\operatorname{Ind} A_{1}$ such that for all $j \in\{1,2, \ldots, t\}$ either $\left(U_{j}, U_{j+1}\right)_{A_{1}} \neq 0$ or $\left(U_{j+1}, U_{j}\right)_{A_{1}} \neq 0$. Similarly, since $X_{2} \underset{P \mathrm{SA}_{2}}{\approx} X_{2}^{\prime}$, there is a sequence $X_{2}=V_{1}, V_{2}, \ldots, V_{t}=X_{2}^{\prime}$ in IndA $A_{2}$ such that for all $j \in\{1,2, \ldots, t\}$ either $\left(V_{j}, V_{j+1}\right)_{A_{2}} \neq 0$ or $\left(V_{j+1}, V_{j}\right)_{A_{2}} \neq 0$ if and only if we have a sequence (with refining sequences if necessary) $X_{1} \otimes X_{2}=U_{1} \otimes V_{1}, U_{2} \otimes V_{2}, \ldots, U_{t} \otimes V_{t}=X_{1}^{\prime} \otimes X_{2}^{\prime}$ such that for all $j \in\{1,2, \ldots, t\}$ either

$$
\left(U_{j} \otimes V_{j}, U_{j+1} \otimes V_{j+1}\right)_{A_{1} \otimes A_{2}} \neq 0 \quad \text { or } \quad\left(U_{j+1} \otimes V_{j+1}, U_{j} \otimes V_{j}\right)_{A_{1} \otimes A_{2}} \neq 0
$$

(by taking the tensor homomorphisms). Therefore, $X_{1} \otimes X_{2} \underset{P S\left(A_{1} \otimes A_{2}\right)}{\approx} X_{1}^{\prime} \otimes X_{2}^{\prime}$.

\section{The pseudoblocks of certain finite dimensional algebras}

Here, we determine the pseudoblocks for some finite dimensional algebras. It turns out that the two concepts; blocks and pseudo-blocks, coincide for all.

\subsection{Semisimple algebras}

It is clear that the two notions; blocks and pseudoblocks, coincide for any finite dimensional semisimple algebra $A$; that is $\operatorname{Ind} A / \underset{P S A}{\approx}=\operatorname{IndA} / \underset{A}{\approx}$.

\subsection{The symmetric group algebra $F S_{3}$}

Let $A=F S_{3}$.

1. If $\operatorname{CharF} \nmid\left|S_{3}\right|$, then $A=F S_{3}$ is semisimple, and so IndA/ $\underset{P S A}{\underset{\widetilde{C}}{\approx}}=\operatorname{IndA} / \underset{A}{\approx}$ as shown above.

2. If $C h a r F=2$, then $A$ has two simple module 1,2 and $\operatorname{Ind} A$ (consists of three indecomposable modules) has the following block distribution: $\underbrace{1,}_{B_{1}}{ }_{1}^{1} \underbrace{2}_{B_{2}}$ which clearly coincides with the pseudoblock distribution.

3. If $C h a r F=3$, then $A$ has two simple modules both of dimension 1; $S_{0}$ (the trivial module) and $S_{1}$ (the sign module), and IndA consists of six indecomposable modules all lie in one Brauer block and are connected by the following sequence of $A$-maps

$$
S_{1} \rightarrow \underset{S_{1}}{S_{0}} \rightarrow \underset{S_{1}}{S_{1}} \rightarrow \underset{S_{0}}{S_{1}} \rightarrow \underset{S_{0}}{S_{1}} \rightarrow \underset{S_{1}}{S_{1}} \rightarrow S_{0} .
$$

Hence, $A=F S_{3}$ has a single pseudoblock in this case. Therefore, we have the following

Theorem 5.1. For $A=F S_{3}$ and in all characteristic of $F$, we have IndA $/ \underset{P S A}{\approx}=\operatorname{IndA} / \underset{A}{\approx}$. 


\subsection{The triangular algebra}

Now take

$$
A=\left\{\left(a_{i j}\right) \in M_{n}(F) \mid a_{i j}=0 ; \forall i>j\right\}=\left\{a=\left(\begin{array}{cccc}
a_{11} & a_{12} & \ldots & a_{1 n} \\
& a_{22} & \ldots & a_{2 n} \\
& & \ddots & \vdots \\
& & & a_{n n}
\end{array}\right) ; a_{i j} \in F\right\}
$$

the algebra of $n \times n$ upper triangular matrices (which is isomorphic to the algebra of lower triangular matrices). Then, $A$ is isomorphic to the path algebra of an equi-oriented quiver of type $A_{n}$. By Gabriel's theorem (see [4, Chapter11]), this quiver has $n(n+1) / 2$ indecomposable modules corresponding to the positive roots of Lie algebra of type $A_{n}$. In fact, $A$ acts on the space of column vectors $U=F^{n}$ by matrix multiplication and

$$
N=\left\{\left(\begin{array}{ccccc}
0 & a_{12} & \cdots & \cdots & a_{1 n} \\
& 0 & a_{23} & \cdots & a_{2 n} \\
& & \ddots & \ddots & \vdots \\
& & & 0 & a_{n-1 n} \\
& & & & 0
\end{array}\right)\right\}=J(A)
$$

the Jacobson radical of $A$, and consequently $A$ has $n$ simple (1-dimensional) representations $\psi_{v}: A \longrightarrow F \quad\left(a \longmapsto a_{v v}\right) ; v=$ $1,2, \ldots, n\left(\psi_{v}\right.$ is an algebra map $\left.\psi_{v}=\psi_{\mu} \Leftrightarrow \nu=\mu\right)$. We also have

$N U=\left\{\left(\begin{array}{c}v_{1} \\ v_{2} \\ \vdots \\ v_{n-1} \\ 0\end{array}\right): v_{i} \in F\right\}$, and $N^{i} U=\left\{\left(\begin{array}{c}v_{1} \\ \vdots \\ v_{n-i} \\ 0 \\ 0\end{array}\right): v_{i} \in F\right\}$, so $U \supset N U \supset N^{2} U \supset \ldots \supset N^{n-1} U \supset 0$ is a composition series with $\operatorname{dim} N^{i-1} U / N^{i} U=1 ; \forall i=1,2, \ldots, n \quad$ and $\quad N^{i-1} U / N^{i} U \cong \psi_{n-i+1}$. Therefore, as $A$-module, $U=F^{n}$ has the following (unique) composition series

$$
\begin{gathered}
U \supset N U \supset N^{2} U \supset \ldots \supset N^{n-1} U \supset 0 \\
\psi_{n} \quad \psi_{n-1} \quad \psi_{n-2} \ldots \psi_{2} \quad \psi_{1} .
\end{gathered}
$$

It follows that the quotient module $U_{i, \alpha}=N^{n-i} U / N^{n-i+\alpha} U$ is a uniserial (hence indecomposable) with the following (unique) composition series

$$
\begin{array}{l|l}
\psi_{i} & N^{n-\alpha} U \\
\psi_{i-1} & N^{n-i+1} U \\
\psi_{i-2} & N^{n-i+2} U \\
\psi_{i-\alpha+1} & N^{n-i+\alpha} U
\end{array}
$$

\section{Figure 5.1}

and hence $U_{i, \alpha}=N^{n-i} U / N^{n-i+\alpha} U ;(i=1,2, \ldots, n$ and $\alpha=1,2, \ldots, i)$ give a complete set of indecomposable $A$-modules. Not that $U_{i, \alpha} \cong U_{j, \beta} \Leftrightarrow i=j \wedge \alpha=\beta$ and $U_{i, 1}=\psi_{i}$. The modules $U_{1,1}, U_{2,2}, \ldots, U_{n, n}$ give a complete set of projective indecomposable $A$-modules. In fact, it is clear that $U_{v, v}=L_{v}=\left\{\left(\begin{array}{ccccc}0 & 0 & a_{1 v} & \ldots & 0 \\ 0 & 0 & a_{2 v} & \ldots & 0 \\ \vdots & \vdots & \vdots & \vdots & \vdots \\ \vdots & \vdots & a_{v v} & \vdots & \vdots \\ 0 & 0 & 0 & 0 & 0\end{array}\right): a_{i v} \in F, i \leq v ; v=1,2, \ldots, n\right\} \triangleleft A$. Note that the composition factors of $U_{v, v}=N^{n-v} U / N^{n} U=N^{n-1} U$ are as follows: 


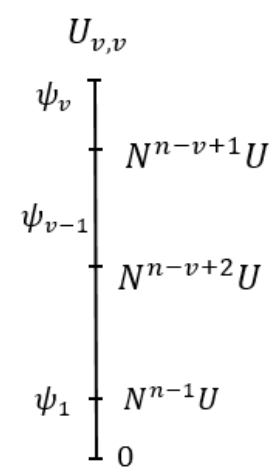

Figure 5.2

The triangular algebra $A$ is not semisimple $(J(A)=N \neq 0)$, hence it has a nontrivial block theory. In fact, $A=\sum_{1 \leq v \leq n}^{\oplus} U_{v, v}$ (projective indecomposable $A$-modules PIM decomposition) is known to be connected; i.e. it has a single non-zero central idempotent, namely $I_{n}$, and so it has a single block. On the other hand, from the structure of the objects $U_{i, \alpha}=N^{n-i} U / N^{n-i+\alpha} U$ $(i=1,2, \ldots, n$ and $\alpha=1,2, \ldots, i)$ of $\operatorname{Ind}(A)$, the objects of the class $\operatorname{Ind}(A)$ can be connected by a series of $A$-maps as follows:

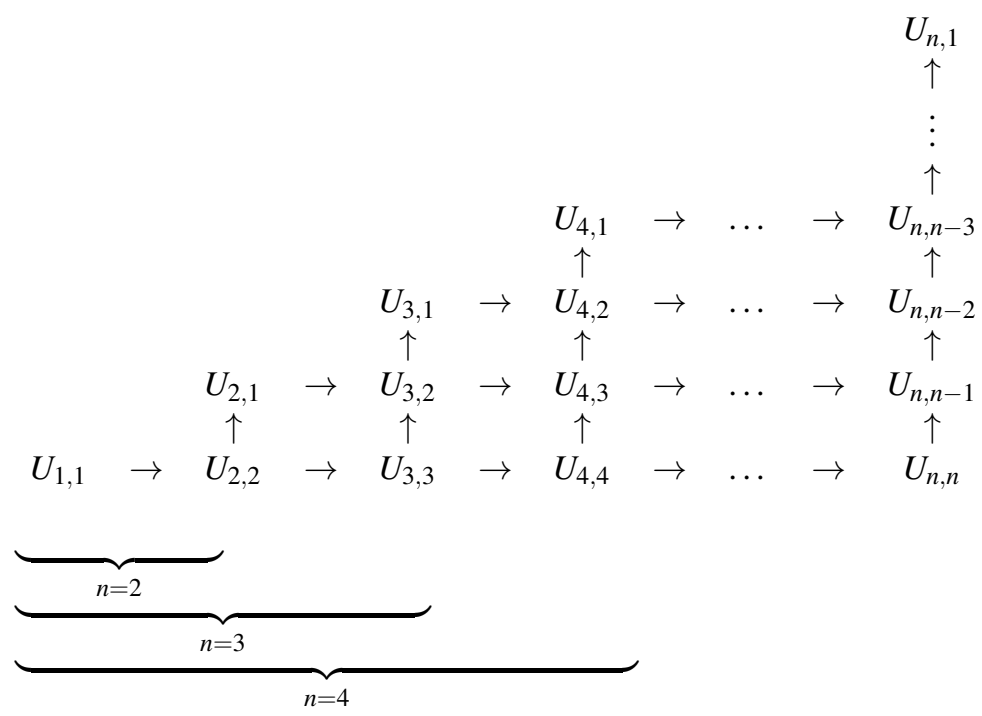

Therefore, $A$ has a single pseudo-block, and so we have:

Theorem 5.2. For the triangular algebra A over a field $F$, we have IndA $\underset{P S A}{\approx}=\operatorname{IndA} / \underset{A}{\approx}$.

\subsection{The group algebra of cylic groups}

We now consider the group algebra of cyclic group $A=F C_{n} ; n=p^{a} e ; p \nmid e$ over a field of characteristic $p$. It is known (see [2], p.34) that $A=F C_{n}$ has $e$ simple (all are 1-dimensional) modules $\left\{S_{\lambda} \mid \lambda\right.$ is an $e$-th root of 1$\}$, where $S_{\lambda}=F$ on which $C_{n}$ acts by multiplication with $\lambda$. It is also known that $A=F C_{n}$ has a total of $n=p^{a} e$ indecomposable modules. For each integer $1 \leq m \leq p^{a}$, there is a uniserial module $L_{\lambda, m}$ of dimension $m$ with all composition factors are isomorphic to $S_{\lambda}$ (note that $L_{\lambda, 1}=S_{\lambda}$ ). The set $\left\{L_{\lambda, m} \mid \lambda, m\right\}$ gives a complete set of $n=p^{a} e$ indecomposable $F C_{n}$-modules. Clearly, PIM $=\left\{L_{\lambda, p^{a}} \mid \lambda\right\}$ $\left(L_{\lambda, p^{a}}=P\left(S_{\lambda}\right)\right.$ is the projective cover of $\left.S_{\lambda}\right)$, and $F C_{n}=\sum_{\lambda}^{\oplus} L_{\lambda, p^{a}}$. The group algebra $F C_{n}$ has $e$ blocks $\left\{B_{\lambda} \mid \lambda\right\}$, where $B_{\lambda}=\left\{L_{\lambda, m} \mid 1 \leq m \leq p^{a}\right\}$. It is clear from the structure of $L_{\lambda, m}$ that $F C_{n}$ has $e$ pseudo-blocks.

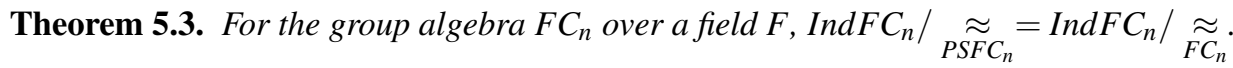

\section{5. $p$-group algebra in characteristic $p$}

The group algebra $F G$ of a finite $p$-group over a field $F$ of characteristic $p$ is known to be indecomposable and has a single simple module, namely the trivial module $1=F_{G}$, and hence has a single block. All indecomposable $F G$-modules are uniserial with all of its composition factors are isomorphic to $F_{G}$. Hence, IndFG forms a single pseudo-block of $F G$.

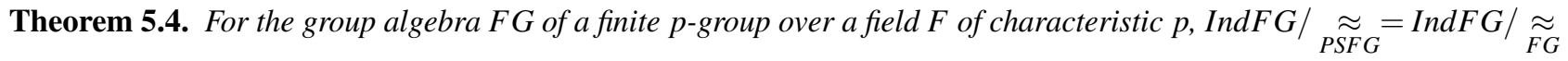




\section{The special linear group $S L(2, p)$}

We now consider the group algebra $A=F S L(2, p)$ in characteristic odd prime number $p$. It is known that $S L(2, p)$ is the only finite group of Lie type which is of finite representation type in the natural characteristic (see [5, Chapter1] ). It is known that $S L(2, p)$ has $p$ ( $p$-regular) conjugacy classes and (hence) $p$ isomorphism classes of simple $F S L(2, p)$-modules of dimensions $1,2,3, \ldots, p$ distributed in three blocks $B_{1}, B_{2}, B_{3}$ (see [6], p.469). We refer to each simple module by its dimension; hence 1 is the natural representation of $S L(2, p)$ and $p$ is the Steinberg representation. There are $p^{2}-p+1$ indecomposable $F S L(2, p)$-modules of which $2 p-1$ of them are either simple or projective (The Steinberg representation is both simple and projective). The number of remaining indecomposable (non-simple non-projective) $F S L(2, p)$-modules is $(p-1)(p-2)$. Denote by $P_{i} ; 1 \leq i \leq p$, the projective cover of the simple $F S L(2, p)$-module $i$. The following theorem describes the structure of the projective indecomposable modules.

Theorem 6.1. [2]. The projective indecomposable FSL(2,p)-modules have the following structures:

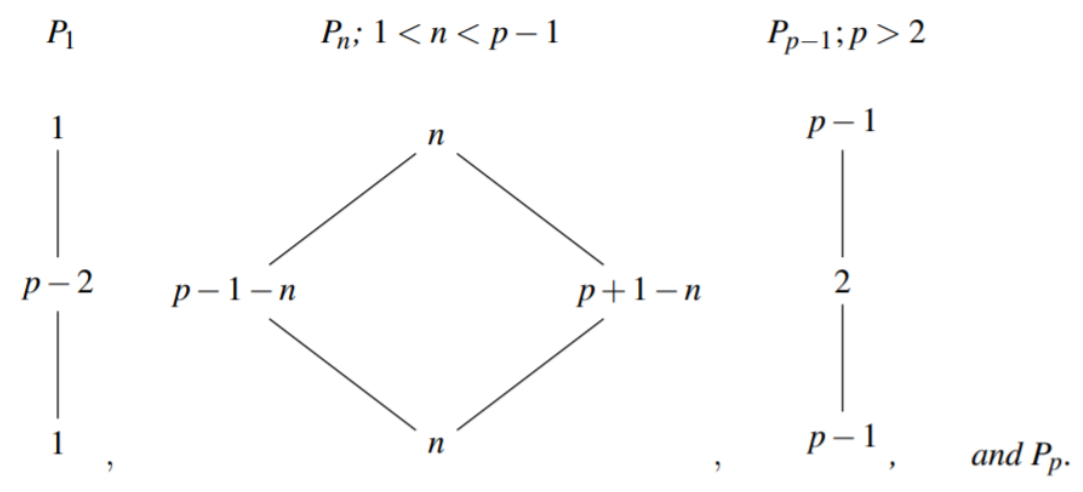

Figure 6.1

The structures of the other indecomposable (non-simple, non-projective) $F S L(2, p)$-modules are explained in the following theorem

Theorem 6.2. [7]. Every (non-simple,non-projective) indecomposable FSL(2,p)-module M has two socle layers. The socle of $M$ consists of the modules $i, i+2, \ldots, j(i \leqslant j)$, and the top consists of the modules $p-j+\varepsilon, p-j+\varepsilon+2, \ldots, p-i+\delta$, where $\varepsilon, \delta= \pm 1$.

The following theorem shows that, the compatiblity between the pseudoblock of $F S L(2, p)$ and block theory.

Theorem 6.3. For the group algebra $A=F G ; G=S L(2, p)$ over a field $F$ of characteristic prime number $p$,

$$
\operatorname{Ind} A / \underset{P S A}{\approx}=\operatorname{Ind} A / \underset{A}{\approx} \text {. }
$$

Proof. First: The block $B_{3}$ (which contains the Steinberg module $p \cong P_{p}$ ) is clearly pseudoblock.

Second: Since $B_{1}$ contains all odd-dimensional simple $A$-modules except $p$, let $P_{m}, P_{i}$ be projective indecomposable $A$ modules, let $m, i$ be simple $A$-modules; for all $m, i \in\{1,3, \ldots, p-2\}$, and let $M_{i}$ be non-simple, non-projective, indecomposable $A$-modules; $i^{\prime}=\{1,2, \ldots, r\}$; in which $P_{m}, P_{i}, m, i$ and $M_{i^{\prime}}$ in $B_{1}$ for all $m, i, i$. Let $P_{i}=i / p-1-i, p+1-i / i$, $P_{m}=m / p-1-m, p+1-m / m, \quad M_{1}=i / p-1-i, p+1-i, \quad M_{2}=p+1-i / i, \quad M_{3}=p+1-m, p-1-m / m$, $M_{4}=p+1-m, p-1-m / i, m, \quad M_{5}=m / p-1-m, p+1-m, \quad M_{6}=p+1-i, p-1-i / i$.

\section{Then, we have six cases as follows:}

1. Let $i, m$ be any two simple $A$-modules. Hence,

$$
i \rightarrow M_{2} \rightarrow m \text {. }
$$

Then, all odd-dimensional simple $A$-modules are connected either ways by a sequence of $A$-module homomorphisms.

2. Let $i, m$ be simple $A$-modules, and let $P_{i}, P_{m}$ be projective indecomposable $A$-modules. Hence,

$$
P_{i} \rightarrow M_{1} \rightarrow i, \quad m \rightarrow M_{3} \rightarrow P_{m}
$$


Then, all odd-dimensional simple $A$-modules and all projective indecomposable $A$-modules are connected either ways by a sequence of $A$-module homomorphisms.

3. Let $M_{i^{\prime}} ; i^{\prime}=\{1,2,3,5\}$ be any non-simple, non-projective, indecomposable $A$-modules, and let $i, m$ be any two simple $A$-modules. Hence,

$$
M_{1} \rightarrow i, \quad M_{2} \rightarrow m, \quad M_{3} \rightarrow P_{m} \rightarrow M_{5} \rightarrow m .
$$

Then, all odd-dimensional simple $A$-modules and all non-simple, non-projective, indecomposable $A$-modules $M_{i^{\prime}} ; i^{\prime}=$ $\{1,2, \ldots, r\}$ are connected either ways by a sequence of $A$-module homomorphisms.

4. Let $P_{i}, P_{m}$ be any two projective indecomposable $A$-modules. Hence,

$$
P_{i} \rightarrow M_{1} \rightarrow i \rightarrow M_{2} \rightarrow p+1-i \rightarrow M_{3} \rightarrow P_{m}
$$

Then, all projective indecomposable $A$-modules $P_{m}, \forall m=\{1,3, \ldots, p-2\}$ are connected either ways by a sequence of A-module homomorphisms.

5. Let $P_{i}, P_{m}$ be any two projective indecomposable $A$-modules, and let $M_{1}, M_{3}, M_{5}, M_{6}$ be non-simple, non-projective, indecomposable $A$-modules. Hence,

$$
P_{i} \rightarrow M_{1}, \quad P_{m} \rightarrow M_{5}
$$

Also,

$$
M_{6} \rightarrow P_{i}, \quad M_{3} \rightarrow P_{m}
$$

Then, all projective indecomposable $A$-modules $P_{m}, \forall m=\{1,3, \ldots, p-2\}$ and all non-simple, non-projective, indecomposable $A$-modules $M_{i} ; i^{\prime}=\{1,2, \ldots, r\}$ are connected either ways by a sequence of $A$-module homomorphisms.

6. Let $M_{1}, M_{2}, M_{3}, M_{4}, M_{5}, M_{6}$ be any non-simple, non-projective, indecomposable $A$-modules. Hence,

$$
\begin{gathered}
M_{6} \rightarrow P_{i} \rightarrow M_{1}, \\
M_{1} \rightarrow i \rightarrow M_{2}, \\
M_{3} \rightarrow P_{m} \rightarrow M_{5},
\end{gathered}
$$

and

$$
M_{4} \rightarrow M_{3}
$$

Then, all non-simple, non-projective, indecomposable $A$-modules are connected either ways by a sequence of $A$-module homomorphisms.

The previous six cases are enough without loss of generality. So, all indecomposable $A$-modules in $B_{1}$ are connected either ways by a sequence of $A$-module homomorphisms as follows:

$$
P_{i} \rightarrow M_{i^{\prime}} \rightarrow i \rightarrow \ldots \leftarrow M_{i^{\prime}}^{\prime} \leftarrow m \leftarrow M_{i}^{\prime \prime} \leftarrow P_{m}
$$

for all $i, m \in\{1,3,5, \ldots, p-2\}$ and $i^{\prime}=\{1,2, \ldots, r\}$.

\section{Thus, the block $B_{1}$ does not split into union of pseudoblocks. So, $B_{1}$ is one pseudoblock.}

Third: Similarly, since the block $B_{2}$ contains all even-dimensional simple $A$-modules.

Let $P_{e}, P_{j}$ be projective indecomposable $A$-modules, let $e, j$ be simple $A$-modules; for all $j, e \in\{2,4, \ldots, p-1\}$, and let $N_{j^{\prime}}$ be non-simple, non-projective, indecomposable $A$-modules; $j^{\prime}=\{1,2, \ldots, r\}$; in which $P_{e}, P_{j}, e, j$, and $N_{j}$ in $B_{2}$ for all $e, j, j^{\prime}$. Let $P_{j}=j / p-1-j, p+1-j / j, \quad P_{e}=e / p-1-e, p+1-e / e, \quad N_{1}=j / p-1-j, p+1-j, \quad N_{2}=p+1-j / j$, $N_{3}=p-1-e, p+1-e / e, \quad N_{4}=p-1-e, p+1-e / e, j, \quad N_{5}=e / p-1-e, p+1-e, \quad N_{6}=p-1-j, p+1-j / j$.

\section{Then, we have six cases as follows:}

1. Let $j, e$ be any two simple $A$-modules. Hence,

$$
j \rightarrow N_{2} \rightarrow e .
$$

Then, all even-dimensional simple $A$-modules are connected either ways by a sequence of $A$-module homomorphisms.

2. Let $j, e$ be simple $A$-modules, and let $P_{j}, P_{e}$ be projective indecomposable $A$-modules. Hence,

$$
P_{j} \rightarrow N_{1} \rightarrow j, \quad e \rightarrow N_{3} \rightarrow P_{e}
$$

Then, all even-dimensional simple $A$-modules and all projective indecomposable $A$-modules are connected either ways by a sequence of $A$-module homomorphisms. 
3. Let $N_{j^{\prime}} ; j^{\prime}=\{1,2,3,5\}$ be any non-simple, non-projective, indecomposable $A$-modules, and let $j, e$ be any two simple $A$-modules. Hence,

$$
N_{1} \rightarrow j, \quad N_{2} \rightarrow e, \quad N_{3} \rightarrow P_{e} \rightarrow N_{5} \rightarrow e .
$$

Then, all even-dimensional simple $A$-modules and all non-simple, non-projective, indecomposable $A$-modules $N_{j^{\prime}} ; j^{\prime}=$ $\{1,2, \ldots, r\}$ are connected either ways by a sequence of $A$-module homomorphisms.

4. Let $P_{j}, P_{e}$ be any two projective indecomposable $A$-modules. Hence,

$$
P_{j} \rightarrow N_{1} \rightarrow j \rightarrow N_{2} \rightarrow p+1-j \rightarrow N_{3} \rightarrow P_{e}
$$

Then, all projective indecomposable $A$-modules $P_{e}, \forall e=\{2,4, \ldots, p-1\}$ are connected either ways by a sequence of $A$-module homomorphisms.

5. Let $P_{j}, P_{e}$ be any two projective indecomposable $A$-modules, and let $N_{1}, N_{3}, N_{5}, N_{6}$ be non-simple, non-projective, indecomposable $A$-modules. Hence,

$$
P_{j} \rightarrow N_{1}, \quad P_{e} \rightarrow N_{5}
$$

Also,

$$
N_{6} \rightarrow P_{j}, \quad N_{3} \rightarrow P_{e}
$$

Then, all projective indecomposable $A$-modules $P_{e} ; \forall e=\{2,4, \ldots, p-1\}$ and all non-simple, non-projective, indecomposable $A$-modules $N_{j^{\prime}} ; j^{\prime}=\{1,2, \ldots, r\}$ are connected either ways by a sequence of $A$-module homomorphisms.

6. Let $N_{1}, N_{2}, N_{3}, N_{4}, N_{5}, N_{6}$ be any non-simple, non-projective, indecomposable $A$-modules. Hence,

$$
\begin{aligned}
& N_{6} \rightarrow P_{j} \rightarrow N_{1}, \\
& N_{1} \rightarrow j \rightarrow N_{2}, \\
& N_{3} \rightarrow P_{e} \rightarrow N_{5},
\end{aligned}
$$

and

$$
N_{4} \rightarrow N_{3}
$$

Then, all non-simple, non-projective, indecomposable $A$-modules are connected either ways by a sequence of $A$-module homomorphisms.

The previous six cases are enough without loss of generality. So, all indecomposable $A$-modules in $B_{2}$ are connected either ways by a sequence of $A$-module homomorphisms as follows:

$$
P_{j} \rightarrow N_{j^{\prime}} \rightarrow j \rightarrow \ldots \leftarrow N_{j^{\prime}}^{\prime} \leftarrow e \leftarrow N_{j^{\prime}}^{\prime \prime} \leftarrow P_{e}
$$

for all $j, e \in\{2,4, \ldots, p-1\}$ and $j^{\prime}=\{1,2, \ldots, r\}$.

Thus, the block $B_{2}$ does not split into union of pseudoblocks. So, $B_{2}$ is one pseudoblock.

Thus, for group algebra $F S L(2, p)$ in characteristic odd prime $p$ the two notions blocks and pseudoblocks coincide.

Example 6.4. If $p=2$, then the representations of $S L(2,2) \cong S_{3}$ in characteristic 2 ; hence the two notions blocks and pseudoblocks coincide as stated in section 5.

If $p=7$, then the following are the indecomposable FSL(2,7)-modules:

- The simple FSL(2,7)-modules are: $\underbrace{1,3,5}_{B_{1}}, \underbrace{2,4,6}_{B_{2}}, \underbrace{7}_{B_{3}}$.

- The projective indecomposable FSL(2,7)-modules are:

$$
1 / 5 / 1, \quad 3 / 3,5 / 3, \quad 5 / 1,3 / 5, \quad 4 / 2,4 / 4, \quad 2 / 4,6 / 2, \quad 6 / 2 / 6, \quad 7 .
$$

- The (non-projective non-simple) indecomposable FSL $(2,7)$-modules are:

$5 / 1, \quad 1 / 5, \quad 3 / 5, \quad 5 / 3, \quad 3 / 3, \quad 3,5 / 3, \quad 3 / 3,51,3 / 5, \quad 5 / 1,3,3,5 / 1,3,5$,

$1,3,5 / 3,5, \quad 1,3,5 / 1,3,5, \quad 3,5 / 1,3, \quad 3,5 / 3,5, \quad 1,3 / 3,5$. (in $B_{1}$ )

$2 / 6, \quad 6 / 2, \quad 4 / 2, \quad 2 / 4, \quad 4 / 4, \quad 4,6 / 2, \quad 2 / 4,6, \quad 2,4 / 4, \quad 4 / 2,4, \quad 2,4 / 2,4,6$,

$2,4,6 / 2,4, \quad 2,4,6 / 2,4,6, \quad 2,4 / 2,4, \quad 2,4 / 4,6, \quad 4,6 / 2,4$. (in $B_{2}$ ) 
The total number of indecomposable modules is $43=7^{2}-7+1$, where $\operatorname{Ext}_{F S L(2,7)}(i, j)$ are 1-dimension for all indecomposable FSL(2,7)-modules as stated in ([5], p.117).

The indecomposable FSL $(2,7)$-modules in $B_{1}$ forms a single pseudoblock via the following sequence of homomorphisms: $3 / 3 \rightarrow 1,3,5 / 1,3,5 \rightarrow 1,3,5 / 3,5 \rightarrow 1,3 / 3,5 \rightarrow 1,3 / 5 \rightarrow 5 / 1,3 / 5 \rightarrow 5 / 1,3 \rightarrow 3,5 / 1,3 \rightarrow 3,5 / 1,3,5 \rightarrow 3,5 / 3,5 \rightarrow 3,5 / 3 \rightarrow$ $3 / 3,5 / 3 \rightarrow 3 / 3,5 \rightarrow 3 / 5 \rightarrow 3 \rightarrow 5 / 3 \rightarrow 5 \rightarrow 1 / 5 \rightarrow 1 \rightarrow 5 / 1 \rightarrow 1 / 5 / 1$.

The indecomposable FSL $(2,7)$-modules in $B_{2}$ forms a single pseudoblock via the following sequence of homomorphisms: $4 / 4 \rightarrow 2,4,6 / 2,4,6 \rightarrow 2,4,6 / 2,4 \rightarrow 4,6 / 2,4 \rightarrow 4,6 / 2 \rightarrow 2 / 4,6 / 2 \rightarrow 2 / 4,6 \rightarrow 2,4 / 4,6 \rightarrow 2,4 / 2,4,6 \rightarrow 2,4 / 2,4 \rightarrow 2,4 / 4 \rightarrow$ $4 / 2,4 / 4 \rightarrow 4 / 2,4 \rightarrow 4 / 2 \rightarrow 4 \rightarrow 2 / 4 \rightarrow 2 \rightarrow 6 / 2 \rightarrow 6 \rightarrow 2 / 6 \rightarrow 6 / 2 / 6$.

\section{Acknowledgement}

The main results of this paper are taken from a dissertation written by the first author as a part of a master degree fulfillments from Umm Al-Qura University. The first author thanks her supervisor Prof. Ahmed A. Khammash for his help and guidance and Taif University for their generous grant.

\section{References}

[1] A. Khammash, The pseudoblocks of endomorphism algebras, Int. Math. Forum, 4(48) (2009), 2363-2368.

[2] J. Alperin, Local Representation Theory: Modular Representations as an Introduction to the Local Representation Theory of Finite Groups, Cambridge University Press, 1986.

[3] A. Khammash, Brauer-fitting correspondence on tensor algebra, Int. J. Algebra, 8(19) (2014), 895-901.

[4] K. Erdmann, T. Holm, Algebras and Representation Theory, Springer, 2018.

[5] J. Humphreys, Modular Representations of Finite Groups of Lie Type, Cambridge University Press, 2006.

[6] L. Dornhoff, Group Representation Theory: Modular Representation Theory, M. Dekker, 1972.

[7] D. Craven, Maximal psl 2 subgroups of exceptional groups of lie type, (2019), arXiv:1610.07469. 